\title{
Isomerization Mechanisms of a Labile Co(II) Octahedral Complex
}

\author{
Fabrice Riblet ${ }^{\S}$, Ghenadie Novitchi ${ }^{\mathrm{b}}$, Lothar Helmª ${ }^{\star}$ Aurélian Gulea ${ }^{\mathrm{b}}$, and André E. Merbach ${ }^{\mathrm{a}}$ \\ $\S$ SCS Poster Prize Winner
}

\begin{abstract}
The labile cobalt (II) complex [Co(TUF $)_{2}(\mathrm{Pic})_{2}$ ] with 4,4,4-trifluoro-1-(2-thienyl)-1,3-butanedione (TUF) bidentate and 4-methylpyridine (Pic) monodentate ligands was studied by variable temperature (VT) ${ }^{19} \mathrm{~F}$ NMR from 184 to $312 \mathrm{~K}$. In contrast to the solid state, all the isomers are present in a $\mathrm{CD}_{2} \mathrm{Cl}_{2}$ solution. Thermodynamic parameters $\left(\Delta \mathrm{H}^{\circ}\right.$ and $\left.\Delta \mathrm{S}^{\circ}\right)$ of each isomer with reference to the trans $(\mathrm{N})$-trans $\left(\mathrm{CF}_{3}\right)$-trans isomer were obtained from the fitting of experimental mole fraction values. A kinetics investigation of the isomerization processes was performed by lineshape analysis of the spectra using the Kubo-Sack formalism. The suggested bidentate bondrupture mechanism involves the breaking of the weakest $\mathrm{Co}-\mathrm{O}$ bond (closest to the $\mathrm{CF}_{3}$ withdrawing group) with the TUF ligand.
\end{abstract}

Keywords: Cobalt(II) $\cdot$ Isomer $\cdot$ Labile $\cdot{ }^{19} \mathrm{~F} \mathrm{NMR} \cdot$ Paramagnetic

\section{Introduction}

The understanding of isomerization processes in labile divalent $3 \mathrm{~d}$-metal complexes is still open to many questions [1-9]. Though dynamic NMR spectroscopy is a well-known tool to investigate rather inert complexes, it has also been successfully employed in stereochemical studies of labile cobalt(ii) complexes in solution [8]. Providing that the electron spin relaxation is at its optimum, NMR spectroscopy can be used to investigate equilibria and kinetics of isomeric forms of paramagnetic complexes in solution. It has been established that the dipolar pseudocontact component is the predominant factor for paramagnetic NMR chemical shift in Co(ii) $\left({ }^{4} T_{l g}\right)$ coordination compounds [8]. The observed chemical shifts are important in respect to the broadening of the resonance lines, leading to well-resolved spectra.

Cobalt(ii) adducts $\left[\mathrm{Co}(\mathrm{AA})_{2}(\mathrm{Py})_{2}\right]$ containing acetylacetonates (AA) and pyridines (Py) exist as several isomers. The total number of possible isomers depends on the symmetry of the ligands. In the case of an axially symmetric monodentate pyri- dine and an asymmetric acetylacetonate, five isomers can be drawn (Fig. 1): three isomers have the pyridine $\mathrm{N}$-atoms in the cis-position and two in the trans-position. In this work we report the ${ }^{19} \mathrm{~F}$ NMR study of the isomerization processes that occur in such a labile cobalt(ii) adduct. The KuboSack formalism was used to simulate the temperature-dependent NMR spectra, allowing conclusions to be drawn on the thermodynamic equilibria and on the kinetics of interconversion between the five isomers.

\footnotetext{
${ }^{*}$ Correspondence: Dr. L. Helm ${ }^{\mathrm{a}}$

Tel.: +41216939876

Fax: +41216939875

E-Mail: lothar.helm@epfl.ch

alnstitute of Chemical Sciences and Engineering

Ecole Polytechnique Federale de Lausanne

EPFL-ISIC-LCIB BCH 3108

$\mathrm{CH}-1015$ Lausanne

bMoldavia State University

Department of Inorganic and Physical Chemistry

Chemistry and Chemical Technology Faculty

MD-2009 Chisinau, Moldavia
}

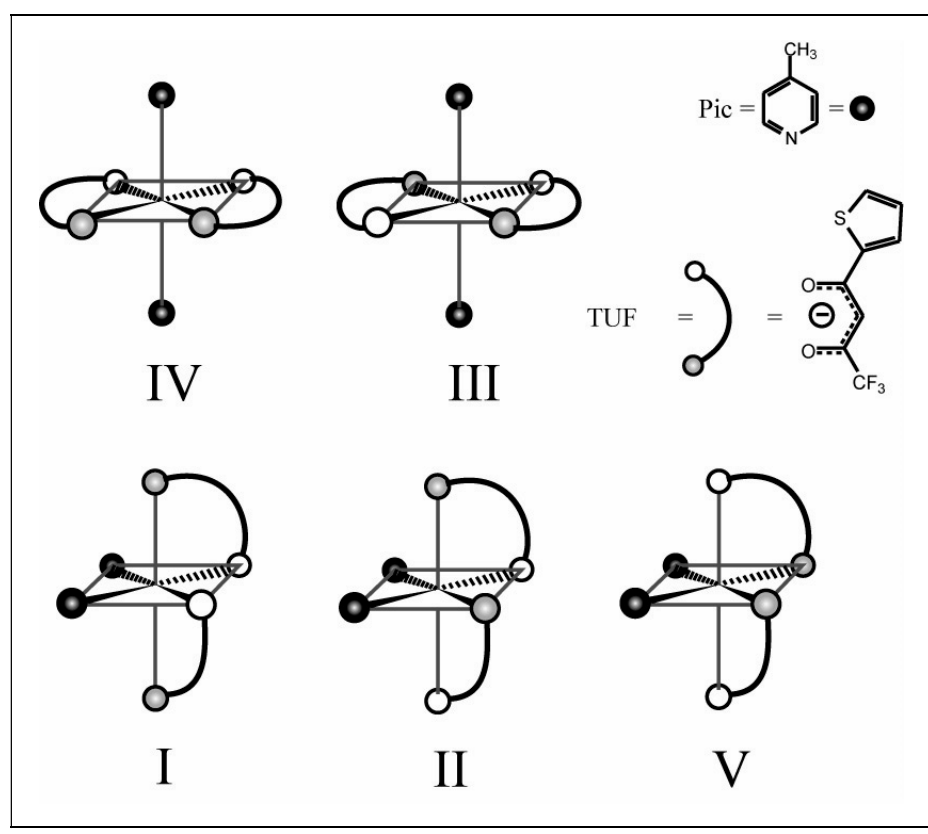

Fig. 1. Schematic representation of the five isomers of compound $\mathbf{1}$ 


\section{Results and Discussion}

\section{Solid State}

As an example we have chosen the complex [Co(TUF $\left.)_{2}(\mathrm{Pic})_{2}\right](\mathbf{1})$, where TUF is 4,4,4-trifluoro-1-(2-thienyl)-1,3-butanedione and Pic, 4-methyl-pyridine. The five isomers were designated by numbers (I-V) as shown in Fig. 1. In the solid state, the isomer $\mathbf{V}$ was observed by X-ray analysis for compound 1 while with another acetylacetonate (tert-butyltrifluoroacetylacetonate) the isomer IV was found [5][9]. Both complexes exhibit a fairly regular octahedral $\mathrm{N}_{2} \mathrm{O}_{4}$ environment formed by two bidentate acetylacetonate and two monodentate picoline ligands. The latter either in cis (such as in $\mathbf{V}$ ) or in trans position (in IV).

\section{Solution Equilibria/VT NMR}

When 1 is dissolved in $\mathrm{CD}_{2} \mathrm{Cl}_{2}$, the ${ }^{19} \mathrm{~F}$ NMR spectrum shows only one broad signal above room temperature (Fig. 2). This signal broadens further with decreasing temperature. Finally, below 260 K, six resonance lines are found. A similar behavior is observed in the ${ }^{1} \mathrm{H}$ NMR spectra. Each single resonance line can be attributed to a symmetrical isomer (as far as the $\mathrm{CF}_{3}$ moieties are concerned i.e. I, III, IV, V) and the two other signals II- $\boldsymbol{a}$ and II- $\boldsymbol{b}$ belong to the less symmetrical one (II). The criteria used for the signal assignment (180-265 K) include comparison of integral ratios, $\operatorname{cis}(\mathrm{N})$ and $\operatorname{trans}(\mathrm{N})$ differentiation in presence of the chiral pyridine [S-(-)-1-(4-pyridyl)ethanol], effect of solvent polarity on the relative stabilities of the isomers and observation of trans influences in a mixture of complexes [9].

The bidentate ligand cannot undergo fast complete dissociation. Therefore its $\mathrm{CF}_{3}$ moiety is an ideal spy to follow the intramolecular isomerization processes A detailed analysis of signal integrals and spectral lineshape simulation using the $\mathrm{Ku}$ bo-Sack formalism led to both thermodynamic and kinetic parameters.

\section{Thermodynamics}

Thermodynamic parameters for the isomerization reactions (Eqn. (1)) are readily obtained from the mole fractions $\left(P_{i}\right)$ of the different isomers as a function of temperature. At equilibrium, the ratios of the mole fractions of the isomers $\left(P_{j} / P_{i}\right)$ are equal to the corresponding relative stability constants $\left(K_{i}, j\right)$ and are related to the thermodynamic parameters through Eqn. (2) and (3).

$$
\text { isomer }-i \quad \rightleftarrows \quad \text { isomer }-j
$$

$$
\Delta G_{i, j}^{\mathrm{o}}=\Delta H_{i, j}^{\mathrm{o}}-T \Delta S_{i, j}^{\mathrm{o}}
$$

$$
\Delta G_{i, j}^{\mathrm{o}}=-R T \ln \frac{P_{j}}{P_{i}}=-R T \ln K_{i, j}
$$

$$
P_{I V}=1-\sum_{i \neq I V} P_{i}
$$

$$
K_{I V, j}=\frac{P_{j}}{P_{I V}}
$$

$$
P_{j}=\left(1-\sum_{i \neq I V} P_{i}\right) \cdot \exp \left(\frac{\Delta S_{i, j}^{\mathrm{o}}}{R}-\frac{\Delta H_{i, j}^{\mathrm{o}}}{R T}\right)
$$

In the slow exchange regime, the mole fractions were directly obtained from the signal integrals. At higher temperature, mole fractions were obtained from the Kubo-Sack treatment (see below). Fitting of Eqn. (5) to the experimental mole fractions, within a wide interval of temperature $(\Delta \mathrm{T}=130 \mathrm{~K})$, led to the thermodynamic parameters. Fig. 3 shows both experimental and calculated mole fractions. Numerical values for isomerization enthalpies and entropies are reported in the Table. The population of isomer $\mathbf{V}$ remains merely constant over the entire temperature range (184-312 K). At low temperature $\operatorname{trans}(\mathrm{N})$ isomers are significantly more stable than $\operatorname{cis}(\mathrm{N})$ ones. The difference in stability of the isomers is less pronounced at increased temperature. At high temperature, isomer II is the most stable isomer; at low temperature it is isomer IV.

\section{Kinetics}

In the NMR slow exchange approximation (i.e. before coalescence of the signals) the transversal relaxation rates can be directly obtained from the linewidth $\mathrm{W}_{1 / 2}$ of each signal (Eqn. (6)). The signals were fitted to Lorentzian functions using a homemade Matlab-based program NMRICMA 3.1.2. [10].

$$
1 / \mathrm{T}_{2}=\pi \mathrm{W}_{1 / 2}
$$

The logarithmic plots of transverse relaxation rates for all five isomers as a function of 1000/T are depicted in Fig. 4 . One can easily observe the two opposing contributions. On the right-hand side (at low temperature), the paramagnetic relaxation is dominating and decreases with temperature. The isomerization is kinetically blocked and the paramagnetic contribution can be determined precisely.

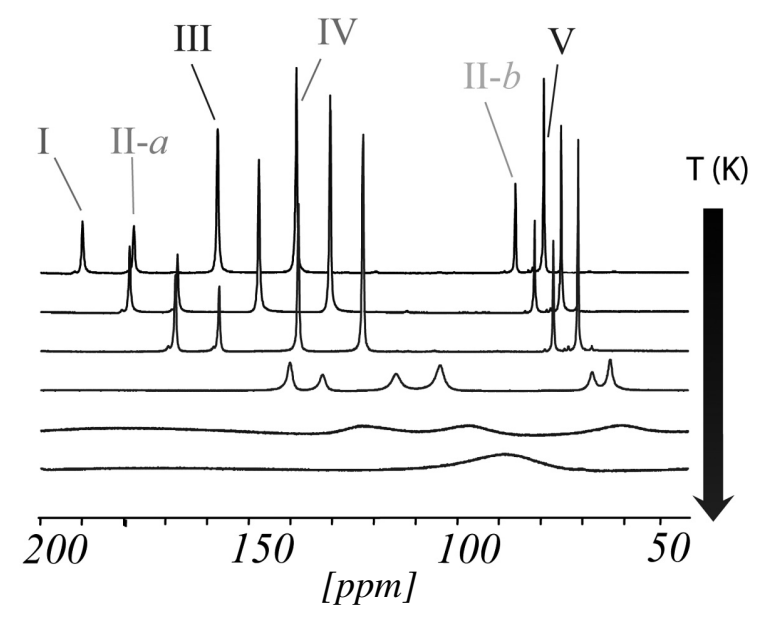

Fig. 2. Evolution of the ${ }^{19} \mathrm{~F} \mathrm{NMR}(375.3 \mathrm{MHz})$ signals of the five isomers of compound $\mathbf{1}\left(25 \mathrm{mM}\right.$ in $\mathbf{1}$ and $175 \mathrm{mM}$ in free $\mathrm{Pic}$, in $\left.\mathrm{CD}_{2} \mathrm{Cl}_{2}\right)$ as a function of temperature (180-310 K)

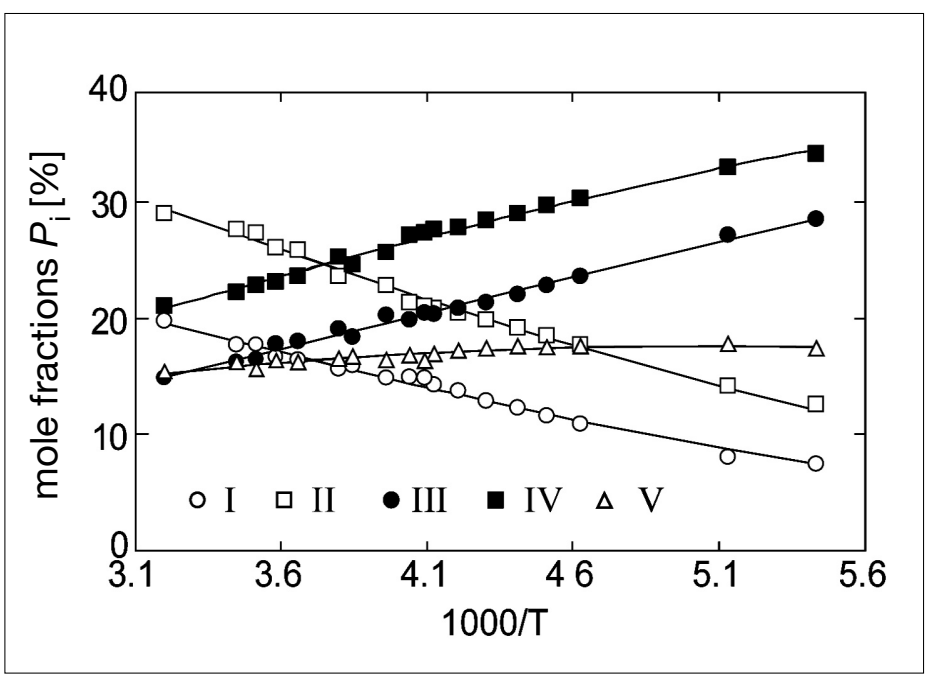

Fig. 3. Experimental and fitted mole fractions of the five isomers of compound 1 in $\mathrm{CD}_{2} \mathrm{Cl}_{2}$ as a function of inverse temperature 
On the left-hand side (high temperature) the kinetic contribution is increasing while the paramagnetic contribution is decreasing and becomes negligible. The kinetic contribution for a specific NMR site is the sum of the rate of isomerization towards the other sites. In this NMR slow exchange regime, the system of equations is therefore the sum of classical Eyring equations for the various isomerization routes plus a typical mono-exponential paramagnetic contribution (Eqn. (7)-(9)). The experimental data for each isomer $(j)$ were simultaneously fitted to those equations using the homemade VISUALISEUR program [11]. In this fit, the total number of isomerization rate constants could be divided by two by taking into account the equilibria constants linking forward and backward isomerization reactions.

$$
\frac{1}{\mathrm{~T}_{2}^{\mathrm{j}}}(\mathrm{T})=\sum_{i} k_{\mathrm{j}>i}(\mathrm{~T})+\frac{1}{\mathrm{~T}_{2}^{\mathrm{para}, \mathrm{j}}}(\mathrm{T})
$$

$$
k_{\mathrm{j}>i}(\mathrm{~T})=\frac{k_{B} T}{h} \exp \left(\frac{\Delta S_{\mathrm{j} i}^{\ddagger}}{R}-\frac{\Delta H_{\mathrm{j}>i}^{\ddagger}}{R T}\right)
$$

$$
\frac{1}{\mathrm{~T}_{2}^{\text {para }, \mathrm{j}}}(\mathrm{T})=\left[\frac{1}{\mathrm{~T}_{2}^{\text {para } \mathrm{j}}}\right]^{\mathrm{T}_{0}} \exp \left[\frac{E_{a \mathrm{j}}}{R}\left(\frac{1}{T}-\frac{1}{T_{0}}\right)\right]
$$

At higher temperatures, the slow exchange approximation is no longer valid and we have used the Kubo-Sack formalism [12] to fit the NMR spectra. Fig. 5 shows a comparison between experimental and calculated spectra at $279.0 \mathrm{~K}$.

Table. Thermodynamic parameters of the isomers of 1 referred to the isomer IV

$\begin{array}{llllll} & \mathrm{I} & \mathrm{II} & \mathrm{III} & \mathrm{IV} & \mathrm{V} \\ \Delta \mathrm{H}_{\mathrm{IV}>\mathrm{j}} / \mathrm{kJ} \mathrm{mol}^{-1} \pm 0.2 & 5.45 & 5.19 & -0.53 & - & 1.38 \\ \Delta \mathrm{S}^{\circ}{ }_{\mathrm{IV}>\mathrm{j}} / \mathrm{Js}^{-1} \mathrm{~mol}^{-1} \pm 0.6 & 16.9 & 19.5 & -4.5 & - & 1.8\end{array}$

\section{Mechanism}

Octahedral cobalt(ii) complexes usually undergo substitution reactions according to dissociative pathways [13]. By analogy, two isomerization patterns involving only single step dissociative mechanisms (pentacoordinated trigonal bipyramidal intermediate) can be suggested:

Monodentate Dissociative Mechanism: ${ }^{1)}$

Dissociative Pic bound/free ligand exchange may proceed with isomerization, or not.

Bidentate Bond-rupture Mechanism: $\left.{ }^{2}\right)$

This involves the breaking of the weakest $\mathrm{Co}-\mathrm{O}$ bond with the TUF ligand (closest to the $\mathrm{CF}_{3}$ withdrawing group). The reformation of the bond may lead to isomerization, or not. This mechanism is not purely dissociative, since TUF remains partially bound to the metal centre in the intermediate. Hence this is a bond-rupture mechanism.

It can be shown that among the ten possible isomerization reactions, only six can directly occur for a single-step dissociative mechanism. These six possible routes are shown in Fig. 6.

Among those six routes, four $(\mathbf{I I} \leftrightarrow$ IV, II $\leftrightarrow \mathbf{I}, \quad \mathbf{I I} \leftrightarrow \mathrm{V}, \quad \mathbf{I I} \leftrightarrow \mathbf{I V}$ and $\mathbf{I I I} \leftrightarrow \mathrm{V})$ are common for both isomerization patterns suggested above. However the route I $\leftrightarrow$ III can only occur for the monodentate dissociative mechanism but not for the bidentate bond-rupture mechanism. In contrary, the route $\mathbf{I I I} \leftrightarrow \mathbf{I V}$ can only occur for the bidentate bond-rupture case but not for the monodentate one. Over the whole range of temperature studied, a very good agreement between experimental and fitted NMR spectra was obtained when the route I $\leftrightarrow$ III was neglected (see the excellent agreement between experimental and calculated spectra in Fig. 5), while neglecting III $\leftrightarrow$ IV did not lead to an acceptable fit of the spectra. On the basis on these observations, the bond rupture mechanism is suggested for the isomerization of compound 1 in $\mathrm{CD}_{2} \mathrm{Cl}_{2}$.

\section{Conclusion and Outlook}

All five possible isomers of the octahedral adduct $\left[\mathrm{Co}(\mathrm{TUF})_{2}(\mathrm{Pic})_{2}\right]$ were observed and identified by ${ }^{19} \mathrm{~F}$ NMR, in $\mathrm{CD}_{2} \mathrm{Cl}_{2}$. The thermodynamic parameters $\left(\Delta \mathrm{H}^{\mathrm{o}}, \Delta \mathrm{S}^{\mathrm{o}}\right)$ between all the isomers for the isomerization equilibria were determined.

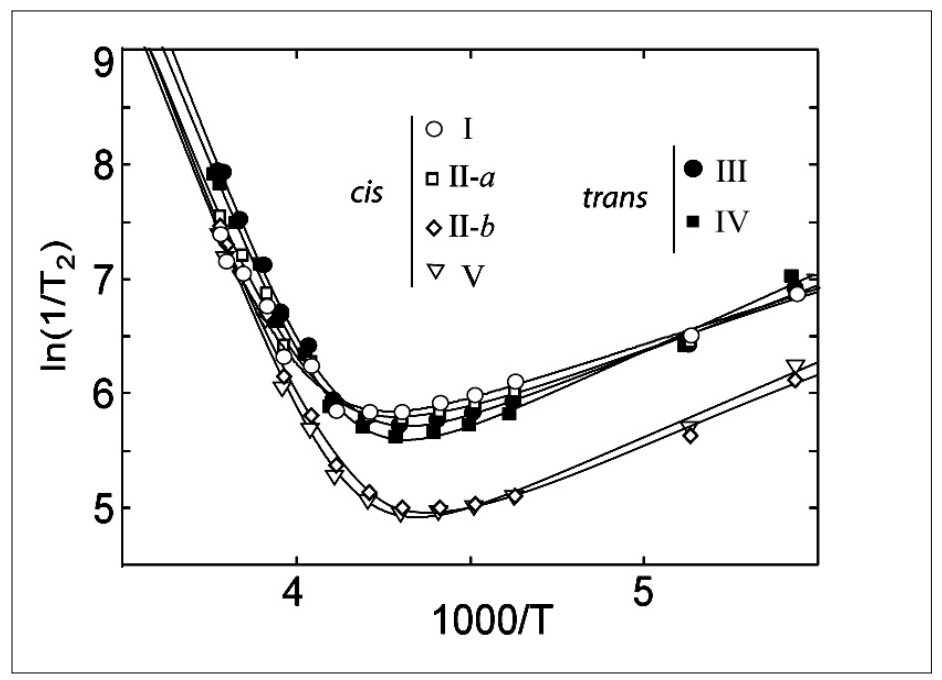

Fig. 4. Evolution of the logarithmic transverse relaxation rates of ${ }^{19} \mathrm{~F} \mathrm{NMR}$ signals of the five isomers of compound 1 in $\mathrm{CD}_{2} \mathrm{Cl}_{2}$ as a function of 1000 / $\mathrm{T}$

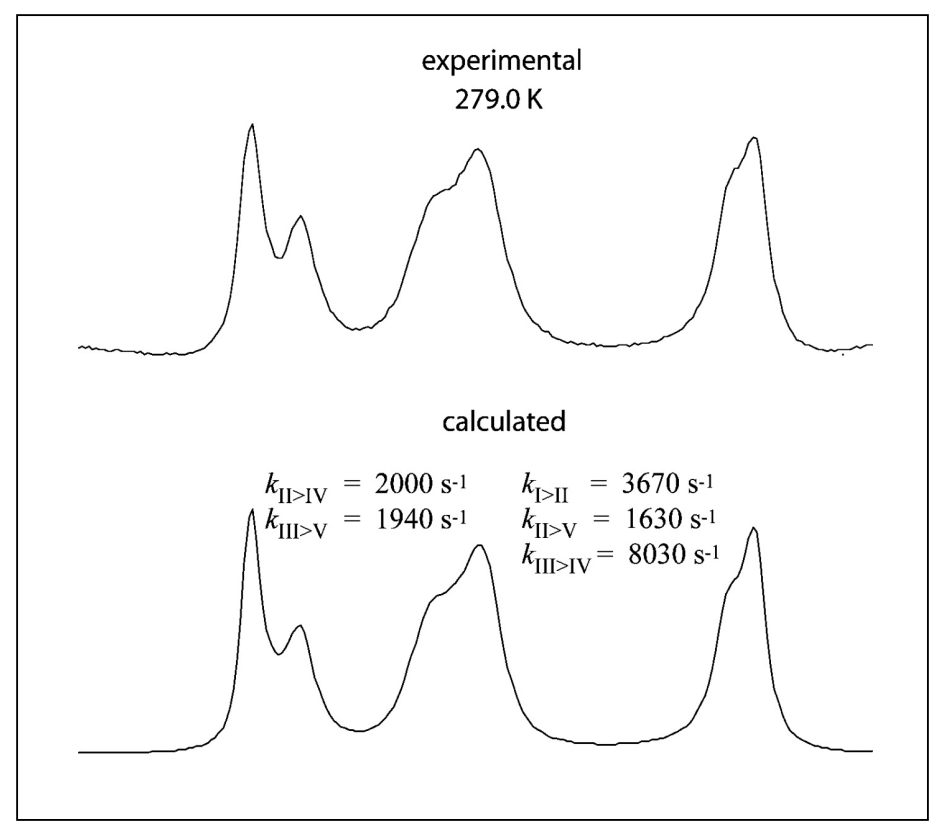

Fig. 5. Experimental (top) compared to calculated (bottom) ${ }^{19} \mathrm{~F} \mathrm{NMR}$ spectra at 279.0K. Isomerization rate constants obtained are indicated $\left(P_{1}=16.6 \%\right.$, $\left.P_{\mathrm{II}}=26.0 \%, P_{\mathrm{III}}=17.8 \%, P_{\mathrm{IV}}=23.1 \%, P_{\mathrm{V}}=16.5 \%\right)$. 


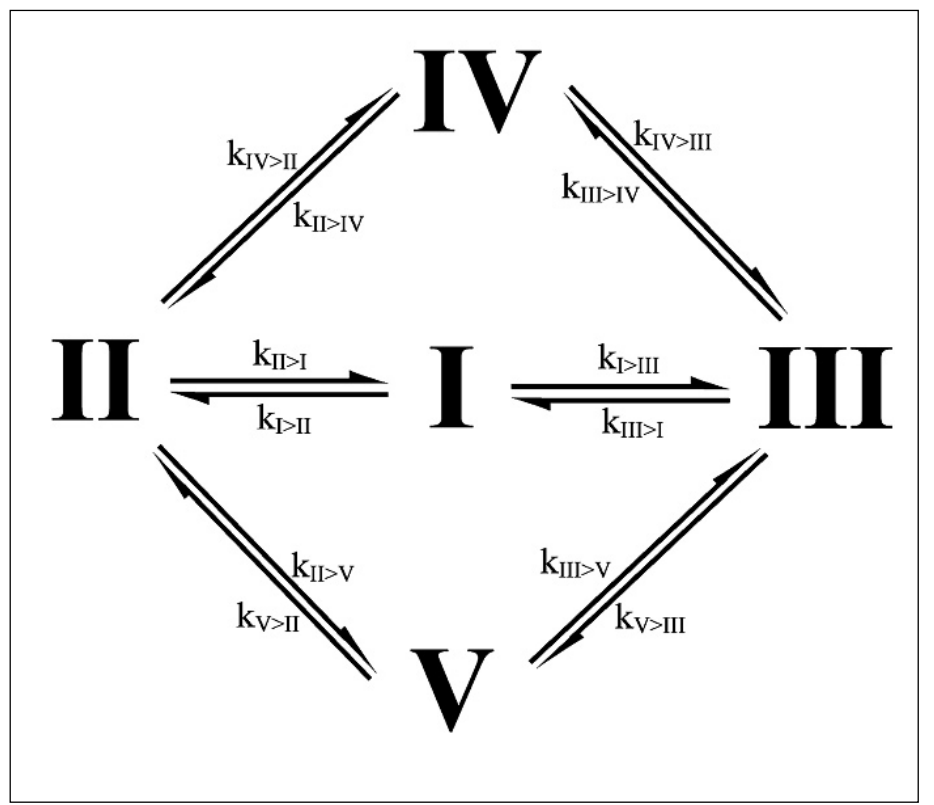

Fig. 6. Possible dissociative isomerization routes for the five isomers of compound 1 in $\mathrm{CD}_{2} \mathrm{Cl}_{2}$

The lineshape analysis of the spectra allowed identification of the isomerization pathways. Among the two possible singlestep dissociative mechanisms, the bidentate single bond-rupture gave the most satisfying fit over the whole temperature range.

The immediate goals pursued are to complement the study of the intramolecular isomerization process by a ${ }^{1} \mathrm{H}$ NMR study of intermolecular monodentate ligand exchange, and to establish volume profiles from variable pressure NMR for a better mechanistic assignment.

Finally, the scope will be extended to other ligands in order to diminish the number of pathways by reducing the number of isomers and obtain a more in-depth view of the isomerization mechanisms of labile Co(ii) adducts.

\section{Acknowledgements}

This work was supported by the Swiss National Science Foundation (G.N.) and the SCOPES (Scientific Cooperation between Eastern Europe and Switzerland) program.

Received: February 19, 2006

[1] K.G. Orrell, Inorg. Chim. Acta 1975, 12, 255.

[2] P. Anstey, K.G. Orrell, J. Chem. Soc. Dalton Trans. 1974, 870.

[3] P. Anstey, K.G. Orrell, J. Chem. Soc. Dalton Trans. 1974, 1771.

[4] A. Gulea, G. Novitchi, S.G. Shova, M.D. Mazus, I. Sandu, Russ. J. Coord. Chem. 1993, 19, 259.

[5] A. Gulea, G. Novitchi, G.A. Timko, N.V. Gerbeleu, Koord. Khim. 1990, 17, 672.

[6] A. Gulea, G. Novitchi, S.G. Shova, I. Sandu, Rev. Roum. Chim. 1993, 38, 505.
[7] A. Gulea, S.G. Shova, G. Novitchi, M.D. Mazus, Russ. J. Coord. Chem. 1994, 20 , 105.

[8] G. Novitchi, A. Chekal, A. Gulea, Russ. $J$. Coord. Chem. 2001, 27, 64.

[9] G. Novitchi, F. Riblet, L. Helm, R. Scopelliti, A. Gulea, A.E. Merbach, Magn. Reson. Chem. 2004, 42, 801.

[10] L. Helm, A. Borel, 'NMRICMA 3.12.', Institut des Sciences et Ingénierie Chimiques, EPFL Lausanne, 2005.

[11] F. Yerly, 'VISUALISEUR-OPTIMISEUR', Institut des Sciences et Ingénierie Chimiques, EPFL Lausanne, 2003.

[12] a) R. Kubo, Nuouo Cimento Suppl. 1957, 6, 1063; b) R.A. Sack, Mol. Phys. 1958, 1, 163; c) C.S. Johnson, Jr., C.G. Moreland, J. Chem. Educ. 1973, 50, 477.

[13] L. Helm, A.E. Merbach, Chem. Rev. 2005 , 105, 1923. 